\title{
Early-type Galaxy Spin Evolution in the Horizon-AGN Simulation
}

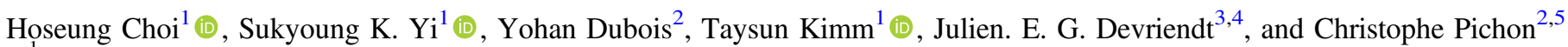 \\ ${ }^{1}$ Department of Astronomy and Yonsei University Observatory, Yonsei University, Seoul 03722, Republic of Korea; choi.h@yonsei.ac.kr, yi@yonsei.ac.kr \\ ${ }^{2}$ Institut d'Astrophysique de Paris, Sorbonne Universités, UMPC Univ Paris 06 et CNRS, UMP 7095 , 98 bis bd Arago, 75014 Paris, France \\ ${ }^{3}$ Dept of Physics, University of Oxford, Keble Road, Oxford OX1 3RH, UK \\ ${ }^{4}$ Universit de Lyon, Universit Lyon 1, ENS de Lyon, CNRS, Centre de Recherche Astrophysique de Lyon UMR5574, F-69230 Saint-Genis-Laval, France \\ ${ }^{5}$ Korea Institute of Advanced Studies (KIAS) 85 Hoegiro, Dongdaemun-gu, Seoul, 02455, Republic of Korea \\ Received 2018 January 7; revised 2018 February 9; accepted 2018 February 15; published 2018 March 29
}

\begin{abstract}
Using the Horizon-AGN simulation data, we study the relative role of mergers and environmental effects in shaping the spin of early-type galaxies (ETGs) after $z \simeq 1$. We follow the spin evolution of 10,037 color-selected ETGs more massive than $10^{10} M_{\odot}$ that are divided into four groups: cluster centrals (3\%), cluster satellites (33\%), group centrals (5\%), and field ETGs (59\%). We find a strong mass dependence of the slow rotator fraction, $f_{\mathrm{SR}}$, and the mean spin of massive ETGs. Although we do not find a clear environmental dependence of $f_{\mathrm{SR}}$, a weak trend is seen in the mean value of the spin parameter driven by the satellite ETGs as they gradually lose their spin as their environment becomes denser. Galaxy mergers appear to be the main cause of total spin changes in $94 \%$ of the central ETGs of halos with $M_{\mathrm{vir}}>10^{12.5} M_{\odot}$, but only $22 \%$ of satellite and field ETGs. We find that non-mergerinduced tidal perturbations better correlate with the galaxy spin down in satellite ETGs than in mergers. Given that the majority of ETGs are not central in dense environments, we conclude that non-merger tidal perturbation effects played a key role in the spin evolution of ETGs observed in the local $(z<1)$ universe.
\end{abstract}

Key words: galaxies: elliptical and lenticular, $\mathrm{cD}$ - galaxies: kinematics and dynamics - galaxies: structure

\section{Introduction}

Massive early-type galaxies (ETGs) are believed to form through numerous mergers and are preferentially found in highdensity environments (the so-called morphology-density relation; Dressler 1980). The importance of mergers in shaping the morphology of galaxies has been well recognized for decades (e.g., Toomre \& Toomre 1972; Barnes 1988; Di Matteo et al. 2007; Dekel et al. 2009; Naab et al. 2014). It was the introduction of integral field unit spectroscopic (IFU) surveys that boosted the investigations on the link between mergers and the properties of their remnants. Notably, the differential rotation of ETGs observed with IFU surveys has drawn much attention (Emsellem et al. 2007; Cappellari et al. 2011). Many theoretical studies immediately followed with scenarios addressing the wide range of observed ETG spins, with a focus on the role of mergers.

Bois et al. (2011) demonstrated that binary merger remnants produced under various merger conditions can reproduce the observed ETG spin-ellipticity distribution (Emsellem et al. 2007). By conducting a set of 44 zoom-in simulations of central galaxies, Naab et al. (2014) claimed that the main driver for the different types of galaxy spins is the difference in their merger histories. Khochfar et al. (2011) took a semi-analytic approach and concluded that massive ETGs that grow predominantly through mergers tend to evolve into slow rotators (SRs).

Studies based on cosmological simulations have shown correlations between galactic spin, ellipticity, and stellar mass, which compare reasonably well to various observations. Lagos et al. $(2017,2018)$ analyzed the EAGLE simulation and found that wet mergers can spin up galaxies, whereas dry mergers tend do the opposite. Other merger properties, such as the merger orbit or the galaxy spin alignment, have been found to be only marginally relevant. On the other hand, based on an analysis of the Illustris simulation data, Penoyre et al. (2017) concluded that it is the reaccretion of cold gas that promotes the galactic spin up, regardless of whether a galaxy undergoes a wet or dry merger. Despite some disparity in the details of such scenarios, it is clear that frequent mergers result in SRs, particularly at low redshifts $(z<2)$, where ETGs have little chance of accreting cold gas.

Because both the stellar mass growth and spin down of ETGs are driven by mergers, it is reasonable to expect a correlation between stellar mass and kinematic properties. In accordance with the scenarios mentioned above, multiple studies have reported a clear dependence of the galactic spin with the stellar mass. In the stellar mass range above $\sim 5 \times 10^{10} M_{\odot}$, the fraction of SRs $\left(f_{\mathrm{SR}}\right)$ increases with the mass from $10 \%$ to $20 \%$ all the way up to over $80 \%$ at $\sim 10^{11.7} M_{\odot}$ (Brough et al. 2017; Greene et al. 2018; van de Sande et al. 2017a; Veale et al. 2017).

Because mergers are one of many environmental effects, it is important to understand the relative significance of the galaxy mass and the local environment. Motivated by the classic morphology-density relation, Cappellari et al. (2011) and Cappellari (2016) suggested a kinematic morphology-density relation that would quantify how ETGs in high-density environments have a lower spin than ETGs in low-density environments. The kinematic morphology-density relation has been supported by the observations of a handful of clusters (Houghton et al. 2012; D'Eugenio et al. 2013; Jimmy et al. 2013; Fogarty et al. 2014; Scott et al. 2014). The dependence of galaxy kinematics on the environment, however, is being challenged. By compiling observations of 22 central galaxies from the literature, Oliva-Altamirano et al. (2017) showed that the dependency of the central galaxy spin on the host halo mass is very weak at $1.8 \sigma$. Moreover, recent studies that are based on more extensive samples of IFU surveys have also claimed that there is only a negligible environmental dependency at a fixed stellar mass, suggesting 
that the apparent kinematic morphology-density relation is simply driven by the mass dependence of ETGs on the environment (Brough et al. 2017; Greene et al. 2017, 2018; Veale et al. 2017).

Nevertheless, the role of the environment may still be important to understand the evolution of ETGs in a wider scope. Unlike massive ETGs, a clear environmental dependence was reported for dwarf ETGs with $M_{*}<5 \times 10^{9} M_{\odot}$ in the Virgo cluster (Guérou et al. 2015; Toloba et al. 2015). It is not surprising that the kinematic transformation of dwarf ETGs is driven through the environmental effects of the large host potential because smaller galaxies are more easily affected by their environment (Boselli et al. 2009, 2014; Vollmer 2009, c.f. Janz et al. 2017). The relative importance of mergers and environmental effects in the mass range between massive ETGs and dwarf ETGs has been discussed in a numerical study by Choi \& Yi (2017). Based on 16 zoom-in hydrodynamic simulations of galaxy clusters, they found that galaxy mergers are the main driver of the spin evolution for the most massive ETGs $\left(M_{*}>5 \times 10^{10} M_{\odot}\right)$, while they cannot fully account for the spin change of intermediate-mass systems $\left(5 \times 10^{9} M_{\odot}<M_{*}<5 \times 10^{10} M_{\odot}\right)$. While the spin of the most massive galaxies is thought to be driven by numerous mergers, more investigations are needed to understand the spin evolution of dwarf and intermediate-mass ETGs and their connections to mergers and environments.

In the present study, we investigated the spin properties of a large number of intermediate- to high-mass ETGs based on the cosmological simulation, Horizon-AGN. We show that (1) mergers are important in shaping the ETG spin, but only for massive central galaxies. (2) Satellite galaxies do have lower spins compared to the field counterparts, (3) and this is due to tidal perturbation in the high-density environments, not the mergers. In Section 2, we describe the simulation data, postprocessing, and derivation of the galaxy properties. The relation between the spin and other galaxy properties in various subsamples is given in Section 3. Finally, we summarize and discuss the results in Section 4.

\section{Methods}

\subsection{Horizon-AGN Simulation}

We used the Horizon-AGN simulation (Dubois et al. 2014), which was performed using the adaptive mesh refinement code RAMSES (Teyssier 2002). The assumed cosmology in the simulation is a flat $\Lambda \mathrm{CDM}$ universe with a Hubble constant of $H_{0}=70.4 \mathrm{~km} \mathrm{~s}^{-1} \mathrm{Mpc}^{-1}$, a baryon density of $\Omega_{b}=0.0456$, a total matter density of $\Omega_{m}=0.272$, a dark energy density of $\Omega_{\Lambda}=0.728$, an rms fluctuation amplitude at $8 h^{-1} \mathrm{Mpc}$ of $\sigma_{8}=0.809$, and a spectral index $n=0.963$, which is consistent with the seven year Wilkinson Microwave Anisotropy Probe analysis (Komatsu et al. 2011). The simulation volume is $\left(100 h^{-1} \mathrm{Mpc}\right)^{3}$ and contains 14 clusters of mass $M_{\text {vir }}>10^{14} M_{\odot}, 367$ groups of mass $10^{13} M_{\odot}<M_{\text {vir }}<$ $10^{14} M_{\odot}$, and field ETGs.

Radiative gas cooling is modeled following Sutherland \& Dopita (1993). A uniform ultraviolet (UV) background heating is activated after $z_{\text {reion }}=10$, based on Haardt \& Madau (1996). Cells with a hydrogen number density above $n_{0}=0.1 \mathrm{H} \mathrm{cm}^{-3}$ are allowed to form stellar particles through a Poisson random process (Rasera \& Teyssier 2006; Dubois \& Teyssier 2008). A $2 \%$ star formation efficiency per free-fall time is assumed in the
Schmidt law $\dot{\rho}=\varepsilon_{*} \rho_{g} / t_{\mathrm{ff}}$, where $\rho_{\mathrm{g}}$ is the gas density, and $t_{\mathrm{ff}}=\sqrt{3 \pi /\left(32 G \rho_{\mathrm{g}}\right)}$ is the local free-fall time (Kennicutt 1998). Stellar feedback from supernova Type Ia, II, and stellar winds is implemented assuming a Salpeter (Salpeter 1955) initial mass function (Kaviraj et al. 2017). The black hole growth is modeled by the Bondi-Hoyle-Lyttleton accretion (Hoyle \& Lyttleton 1939; Bondi \& Hoyle 1944). When the gas accretion rate is low, the black hole launches bipolar jets (radio mode), whereas active galactic nuclei deposit thermal energy isotropically when the accretion rate is high (quasar mode; Dubois et al. 2012).

\subsection{Galaxy Identification and Merger Tree}

The galaxies in the simulation were identified using HaloMaker through the AdaptaHOP method (Aubert et al. 2004), with the most massive sub-node mode (Tweed et al. 2009) applied for stellar particles. A minimum of 64 stellar particles, or $M_{*}=5 \times 10^{7} M_{\odot}$, were used to define a galaxy, but we limit our spin analysis to galaxies more massive than $M_{*}=10^{10} M_{\odot}$ to ensure robust measurements. The smaller galaxies identified are only considered as merging satellites or sources of tidal perturbation in the later part of the analysis.

To determine the size of the galaxies, we first measured the stellar mass above the surface density cut $\left(\Sigma_{\mathrm{M}}>10^{6} M_{\odot} \mathrm{kpc}^{-2}\right)$, which is used to compute a tentative half-mass-radius $\left(R_{\text {eff,ten }}\right)$. The threshold is chosen so that it reasonably covers the entire regions of isolated galaxies. We then recalculated the half-massradius $\left(R_{\text {eff }}\right)$ using the star particles within $R \leqslant 4 R_{\text {eff,ten }}$ and determined the total stellar mass by integrating the mass of star particles within $R \leqslant 4 R_{\text {eff }}$.

To follow the evolution of the galaxy spin, we constructed galaxy merger trees using 787 snapshots of the Horizon-AGN simulation. The time interval between snapshots corresponds to $\Delta a_{\text {exp }} \approx 0.0001$, or 17 Myr. Note that the number of snapshots is larger than usual for cosmological simulation data sets, allowing us to precisely determine the progenitor-descendant relation and to monitor the beginning and end of the mergers.

We defined the beginning of a merger as when a satellite galaxy crosses three times the sum of the radii of the host and satellite galaxies. We also required the orbital angular momentum of the satellite galaxy to stay below the initial value at three times the sum of the radii. If the satellite reacquires the orbital angular momentum or moves outside the radial distance limit, then the merger is considered to exert no effect on the host galaxy in the meantime. The end of a merger is defined as the time at which the satellite is no longer detected by HaloMaker. By filtering out fly-by-like interactions in the very early stages of the minor mergers, we determined the merger stages where the stellar component of the host galaxy actually feels disturbances. All mergers above a merger mass ratio of 1:50 are considered, and mergers with a mass ratio larger than 1:4 are considered as major mergers.

\subsection{Spin Parameter}

To compute the galactic spin parameter $\left(\lambda_{\mathrm{R}}\right.$; Emsellem et al. 2007) of the simulated galaxies, we first generated twodimensional projection maps of the stellar luminosity, the luminosity-weighted line-of-sight velocity, and the luminosityweighted velocity dispersion along the $z$-direction of the simulation. To ensure statistical reliability, we split each stellar 
particle into 60 pseudo particles, which were distributed following a Gaussian kernel with a standard deviation of $0.3 \mathrm{kpc}$. Pseudo particles were grouped using the Voronoi binning technique (Cappellari \& Copin 2003) to achieve a uniform statistical significance over the measurement points. We used the publicly available Multi-Gaussian Expansion (MGE) package (Emsellem et al. 1994; Cappellari 2002) to determine the optical center of a galaxy and measure the ellipticity $\varepsilon(\equiv 1-b / a)$, where $a$ and $b$ are the semimajor and semiminor axis of the fitted ellipse, respectively. We adopted the ellipticity $\varepsilon$ at $\sqrt{a b} \sim R_{\text {eff }}$ as the representative $\varepsilon$ of a galaxy throughout this work. The center of velocity is computed by the mean velocity of the $10 \%$ closest points to the optical center.

The spin parameter of a galaxy was measured following the definition of Emsellem et al. (2007) as

$$
\lambda_{R}=\frac{\sum_{i} F_{i} R_{i}\left|V_{i}\right|}{\sum_{i} F_{i} R_{i} \sqrt{V_{i}^{2}+\sigma_{i}^{2}}},
$$

where $R_{i}$ is the radius of the concentric ellipse, $F_{i}$ is the dustattenuated flux, $V_{i}$ is the luminosity-weighted mean line-ofsight velocity of stellar particles, and $\sigma_{i}$ is the line-of-sight velocity dispersion of the $i$ th spatial bin. Note that we adopted the spin parameter measured within the fitted ellipse of $x^{2} / a^{2}+y^{2} / b^{2}=1$ for a fair comparison with the observations. Figure 2 shows the examples of the spin measurement for randomly selected galaxies of different morphologies and environments (see Section 3.2).

\subsection{Early-type Galaxy Sample}

We defined ETGs to be red galaxies ${ }^{6}$ following Gabor et al. (2010), as

$$
g-r>-0.0042 M_{r}+0.5,
$$

where $M_{r}$ is the Sloan Digital Sky Survey (SDSS) $r$-band absolute magnitude. We calculated the galaxy's $g-r$ color by generating mock images using the stellar population model of Bruzual \& Charlot (2003), assuming a Salpeter initial mass function. Note that the dust extinction is not taken into account during the sample selection procedure to avoid possible contamination by dust-reddened late-type galaxies. This results in 10,037 ETGs out of the total 21,486 galaxies above $M_{*}=10^{10} M_{\odot}(\sim 45 \%$, Figure 1$)$. The fraction of ETGs is comparable to the observations (Skibba et al. 2009; Khim et al. 2015, for example). As a sanity check, we also performed a visual inspection of a randomly selected subsample of $10 \%$ of the total Horizon-AGN galaxies and found that late-type galaxies are very rarely included in our ETG sample. We also checked that using a different selection criterion (Schawinski et al. 2014) does not change our main results.

Figure 2 shows the examples of the spin measurement for randomly selected galaxies of different morphologies and

\footnotetext{
6 Another possible way to classify the morphology of simulated galaxies is based on the orbital eccentricity of the stellar particles and their direction with respect to the galaxy spin (Abadi et al. 2003; Scannapieco et al. 2009; Sales et al. 2012). However, relating the kinematic classification to the observational classification has ambiguities of its own (Bottrell et al. 2017). Thus, we took a simpler approach based on the color-magnitude diagram.
}

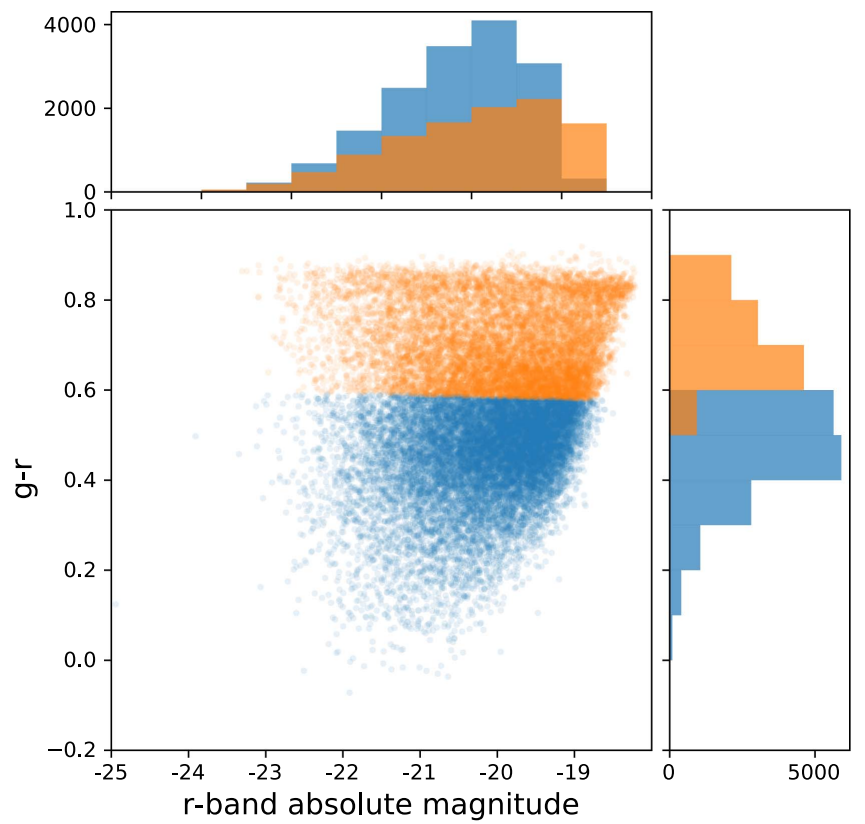

Figure 1. Classification of ETGs based on the color-magnitude diagram (Gabor et al. 2010). Orange dots indicate ETGs, while late types are shown as blue. Note that dust attenuation is not taken into consideration during the sample selection procedure to avoid contamination by dust-reddened late-type galaxies.

environments (see Section 3.2). Also included is the spin parameter as a function of the radius.

\section{Results}

\subsection{Mass and Environmental Dependence of the Whole Sample}

The distribution of spin parameter $\lambda_{\mathrm{R}}$ of the 10,037 ETGs is compared to the ATLAS ${ }^{3 \mathrm{D}}$ observations (Emsellem et al. 2011) in Figure 3(a). The overall shapes of the two distributions are qualitatively similar. With a sufficient number of galaxies, the simulated galaxy distribution appears smooth and displays a single peak at $\lambda_{\mathrm{R}} \approx 0.15$ and $\varepsilon \approx 0.1$. If the demarcation $\lambda_{R}=0.31 \sqrt{\epsilon}$ of Emsellem et al. (2011; dashed black line) is used, then the slow rotator fraction $f_{\mathrm{SR}}$ is $11.2 \%$ (1124/ 10,037): a value that is comparable to $14 \% \pm 2 \%$ according to ATLAS $^{3 \mathrm{D}}$ (Emsellem et al. 2011).

The top and bottom $20 \%$ of the sample galaxy, based on their stellar mass, are presented in Figures 3(b) and (c), respectively. A substantial fraction (30.5\%) of massive ETGs were found to be SRs, whereas only $4.2 \%$ of low-mass ETGs were found to be so. The range of $\varepsilon$ and $\lambda_{\mathrm{R}}$ values represented in Figures 3(b) and (c) is different. Massive ETGs form a broad peak at $\varepsilon \approx 0.1, \lambda_{\mathrm{R}} \approx 0.1$ with notable scatters in both $\varepsilon$ and $\lambda_{\mathrm{R}}$ axes. By contrast, low-mass ETGs are clustered tightly to form an elongated peak from $\varepsilon \approx 0.1, \lambda_{\mathrm{R}} \approx 0.2$ to $\varepsilon \approx 0.2, \lambda_{\mathrm{R}} \approx 0.45$. Some SRs were found to have very high ellipticities with complex kinematic structures inside $1 R_{\text {eff }}$ owing to satellite remnants (fourth galaxy in Figure 2, for example), which is a result corroborated by Naab et al. (2014), Cappellari (2016), and van de Sande et al. (2017b).

The correlation between stellar mass and $f_{\mathrm{SR}}$ is clearly visible, i.e., massive galaxies are preferentially SRs (Figure 4(a)). However, the trend in mean $\lambda_{\mathrm{R}}$ against stellar mass is weaker than $f_{\mathrm{SR}}$ owing to the large scatter in $\lambda_{\mathrm{R}}$ (Figure 4(b)). The scatters are markedly larger in low stellar mass bins compared to 

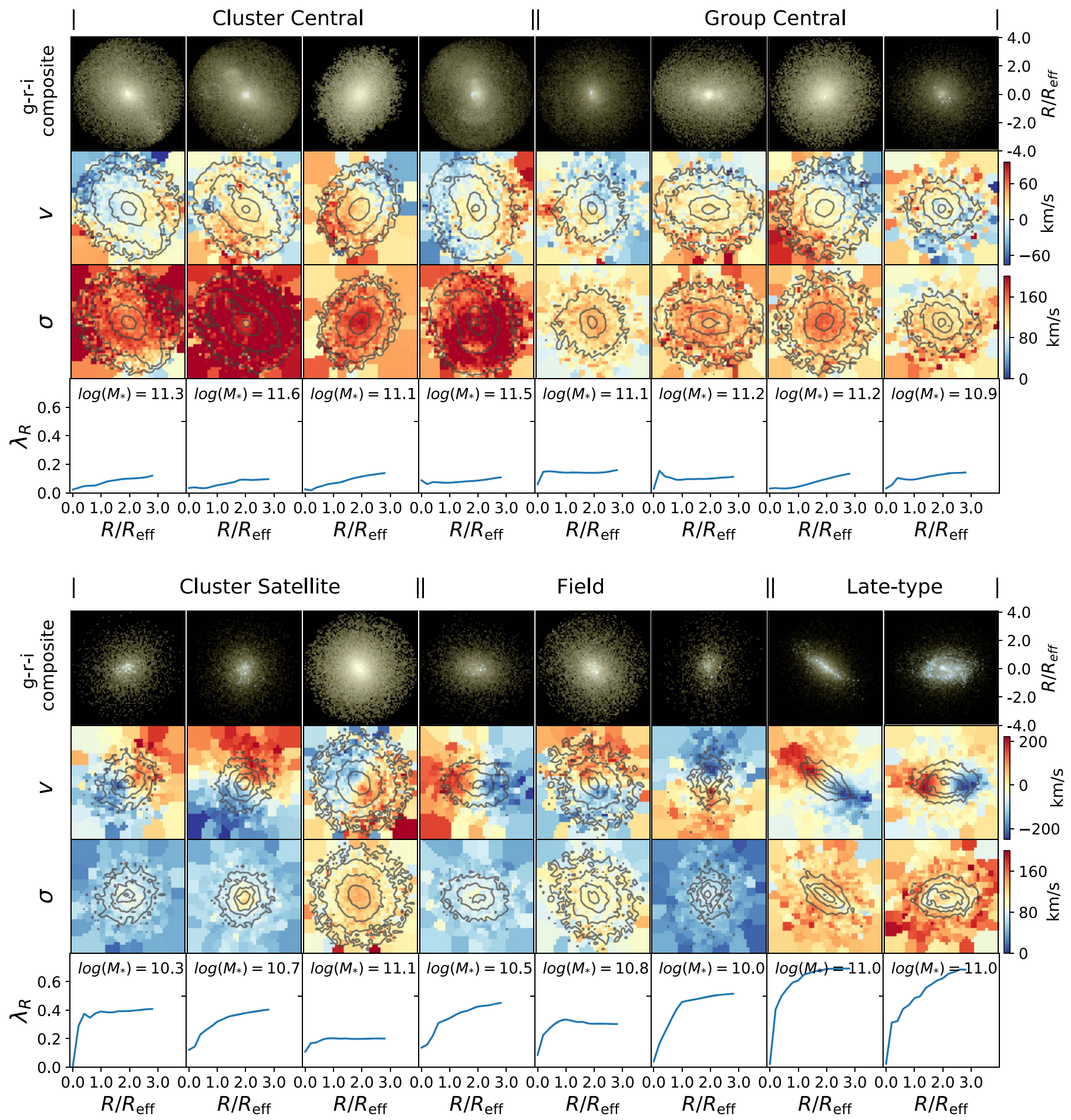

Figure 2. Examples of 14 ETGs and 2 late-type galaxies from Horizon-AGN. From the top to bottom, each row shows the composite image of the SDSS $g, r$, and $i$ bands, line-of-sight velocity, line-of-sight velocity dispersion, and radial profile of $\lambda_{\mathrm{R}}$ of each galaxy. The last two late-type galaxies are not included in our ETG sample, but presented for comparison purposes.

high stellar mass bins, suggesting that the same process responsible for a galaxy spin down may also be responsible for a mass growth of massive galaxies. Alternatively, the significant scatters in $\lambda_{\mathrm{R}}$ of low-mass bins imply that processes unrelated to mass growth are operating to shape galactic spin.

Next, we examined the relationship between $\lambda_{\mathrm{R}}$ and the galaxy's local environmental density $D_{5}$, which is defined as the distance to the fifth closest galaxy more massive than
$10^{10} M_{\odot}$. The trends in both the $\lambda_{\mathrm{R}}$ distribution (Figure 4(c)) and $f_{\mathrm{SR}}$ (Figure $4(\mathrm{~d})$ ) are not as pronounced compared to the mass dependency. Only a weak dependence of mean $\lambda_{\mathrm{R}}$ on the local density below $D_{5} \lesssim 3 \mathrm{Mpc}$ was found, and no trend is evident in $f_{\mathrm{SR}}$.

To disentangle the environmental dependence from the mass dependence, we further split the sample into four mass bins in Figure 5. A mild correlation between $\lambda_{\mathrm{R}}$ and $D_{5}$ is found, with 


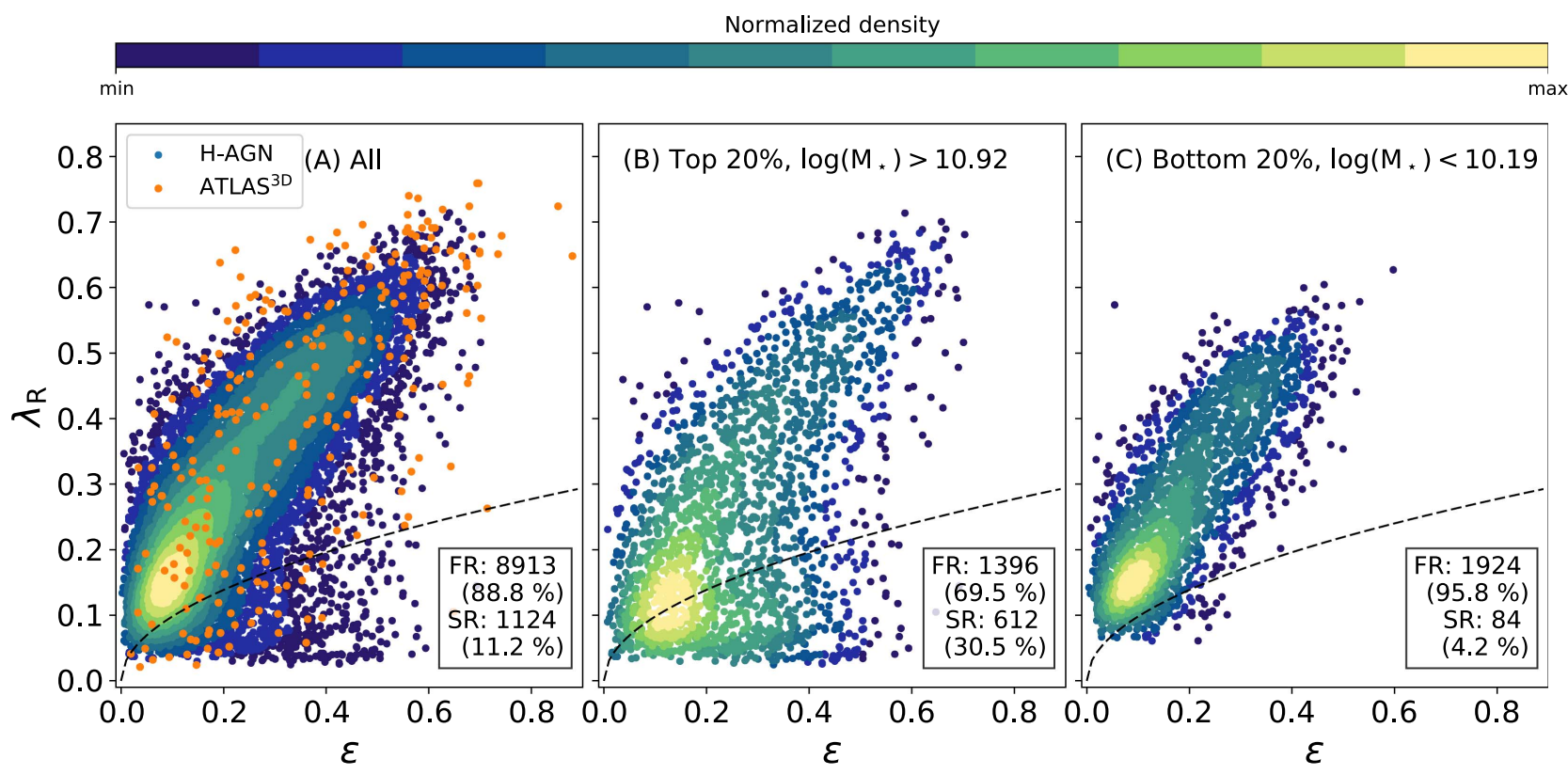

Figure 3. Spin parameter $\lambda_{R}$ vs. ellipticity $\varepsilon$ for all sample ETGs (panel a, left). Orange points indicates 260 ETGs from ATLAS ${ }^{3 D}$. The 10 step shades represent a kernel density estimation of the galaxy distribution normalized in each panel. Slow rotators are defined by the demarcation line at $R_{e}$ from Emsellem et al. (2011). The middle panel shows the distribution for the top $20 \%$ in mass $\left(M_{*}>8.1 \times 10^{10} M_{\odot}\right)$ (panel b), while the bottom $20 \%$ in mass $\left(M_{*}<1.5 \times 10^{10} M_{\odot}\right)$ is shown in the right panel (panel c).

the exception of the most massive bin (Figure 5(a)). However, the $f_{\mathrm{SR}}$ in each of the mass bins shows no evidence for trends (Figure 5(b)), which is a result that is consistent with the findings of Brough et al. (2017) and Greene et al. (2017). We argue that the discrepancy between the mean $\lambda_{\mathrm{R}}$ and $f_{\mathrm{SR}}$ demonstrates that $f_{\mathrm{SR}}$ is only sensitive to very slow-rotating ETGs and fails to capture the entire picture.

\subsection{Environmental Dependence by Group Classification}

In order to better understand the environmental dependence of spin, we split our ETG sample into four subsets: cluster central, cluster satellite, group central, and field ETGs. A group in this regard includes clusters, and thus if the direct host halo of a galaxy exceeds $M_{\text {vir }}>10^{13} M_{\odot}$, we classify it as a cluster central. Cluster satellites are satellite ETGs of halos with $M_{\text {vir }}>10^{13} M_{\odot}$. If the host halo of a galaxy is within the mass range of $10^{12.5} M_{\odot}<M_{\text {vir }}<10^{13} M_{\odot}$, we categorize it as a group central galaxy. Finally, the remaining galaxies (satellites of halos $M_{\mathrm{vir}}<10^{13} M_{\odot}$ and centrals of halos $M_{\mathrm{vir}}<$ $10^{12.5} M_{\odot}$ ) are defined as field ETGs. Below the halo mass of $M_{\text {vir }}<10^{13} M_{\odot}$, satellites do not have a sufficient mass difference to the host halo because only the galaxies more massive than $M_{*}=10^{10} M_{\odot}$ are considered. There are 57 $(0.95 \%)$ such satellite ETGs in the field ETG group. Additionally, below the halo mass of $M_{\mathrm{vir}}<10^{12.5} M_{\odot}$, central galaxies are not dominated by mergers. For this reason, we found this mass cut best represents the differential spin evolutions of central and satellite galaxies owing to their varying dependence on mergers and environmental effects.

We found 267 cluster centrals, 3,234 cluster satellites, 475 group centrals, and 5721 field ETGs. Note that, owing to our selection criterion of the ETG sample based on the colormagnitude diagram (see Figure 1), the field ETG sample may include a small fraction of late-type galaxies (LTGs). Thus, we consider field ETGs to represent a control sample of cluster satellites with similar properties except for their environments.

Figure 6 shows the correlation between the mean $\lambda_{\mathrm{R}}$ and $D_{5}$ for each subsample according to the division of each group into four same-sized mass bins. The cluster centrals are consistently rotating slowly throughout all environments (Figure 6(a)), whereas the mean $\lambda_{\mathrm{R}}$ of cluster satellites in Figure 6(b) demonstrate decreasing trends toward denser environments in all mass bins, especially in the dense region with $D_{5}<1 \mathrm{Mpc}$ (Figure 6(b)). The group central ETGs comprise a combination of both high-mass, slow-rotating ETGs, and low-mass, fastrotating ETGs (Figure 6(c)), implying this is where the transitions of the central galaxies from FR to SR is operating. Lastly, the field ETGs all exhibit fast rotation at mean $\lambda_{\mathrm{R}} \approx 0.35$ regardless of their stellar masses or environments (Figure 6(d)).

When combined, cluster centrals and group centrals form a continuous distribution. They are similar in mass (group centrals are slightly less massive) and uniformly slow-rotating (group centrals rotate slightly faster). The same is true for cluster satellites and field ETGs; both share similar mass ranges and their $\lambda_{\mathrm{R}}$ distribution are continuous. Taken together, centrality appears to be a major factor in determining the spin of ETGs.

A number of other findings transpire from our measurements. First, satellites have lower spins than field ETGs, with decreasing $\lambda_{\mathrm{R}}$ toward denser environments. Second, smaller satellite ETGs have a lower spin. This trend is not visible in field ETGs, and is opposite to the trend observed for the entire ETG sample (see Figure 5). These differences indicate that the factors responsible for spin loss likely differs from those operating to influence the mass growth of cluster satellites.

It is notable that cluster satellites alone (in Figure 6(b)) appear to show some environmental dependence in addition to the mass dependence. We find that this is in reasonable 

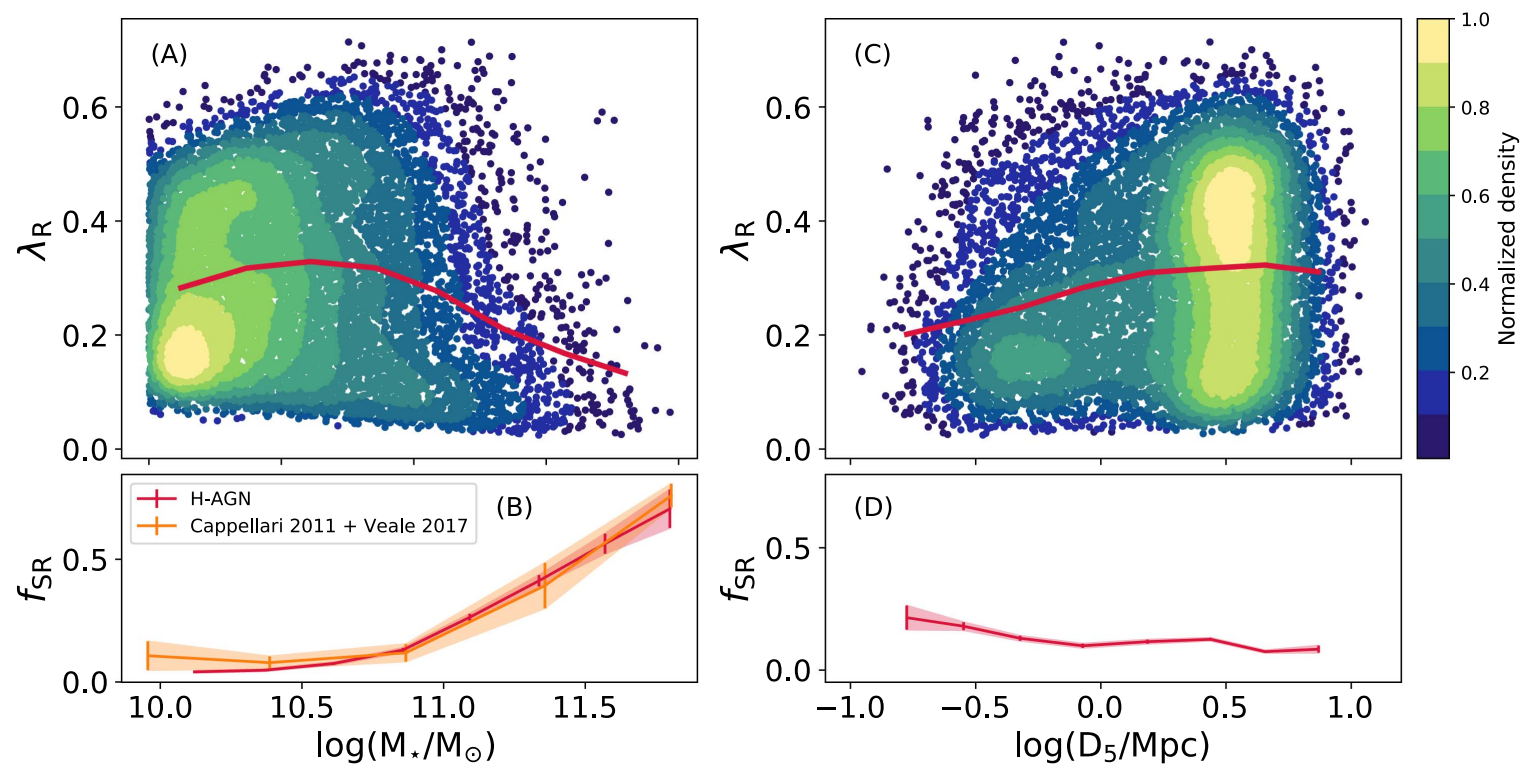

Figure 4. Comparison of $\lambda_{R}$ dependence on mass (left) and environment (right). The top panels show the distribution of $\lambda_{R}$ with galaxy stellar mass. The 10-step shades represent a kernel density estimation of the galaxy distribution. The red solid line denotes the mean spin parameter measured. In the bottom left panel, we present the dependence of the fraction of SRs on stellar mass, which is largely consistent with observations (Greene et al. 2017; Oliva-Altamirano et al. 2017; van de Sande et al. 2017a; Veale et al. 2017). As shown, the most of the massive galaxies are concentrated in a low $\lambda_{\mathrm{R}}$ region, whereas the scatter increases significantly for less massive ETGs. Compared to the mass dependency of $f_{\mathrm{SR}}$, the environmental dependency of $f_{\mathrm{SR}}$ is only marginal. Nonetheless, the mean $\lambda_{\mathrm{R}}$ of the galaxies in very dense regions $\left(\mathrm{D}_{5}<1 \mathrm{Mpc}\right)$ shows a weakly positive correlation with $D_{5}$.
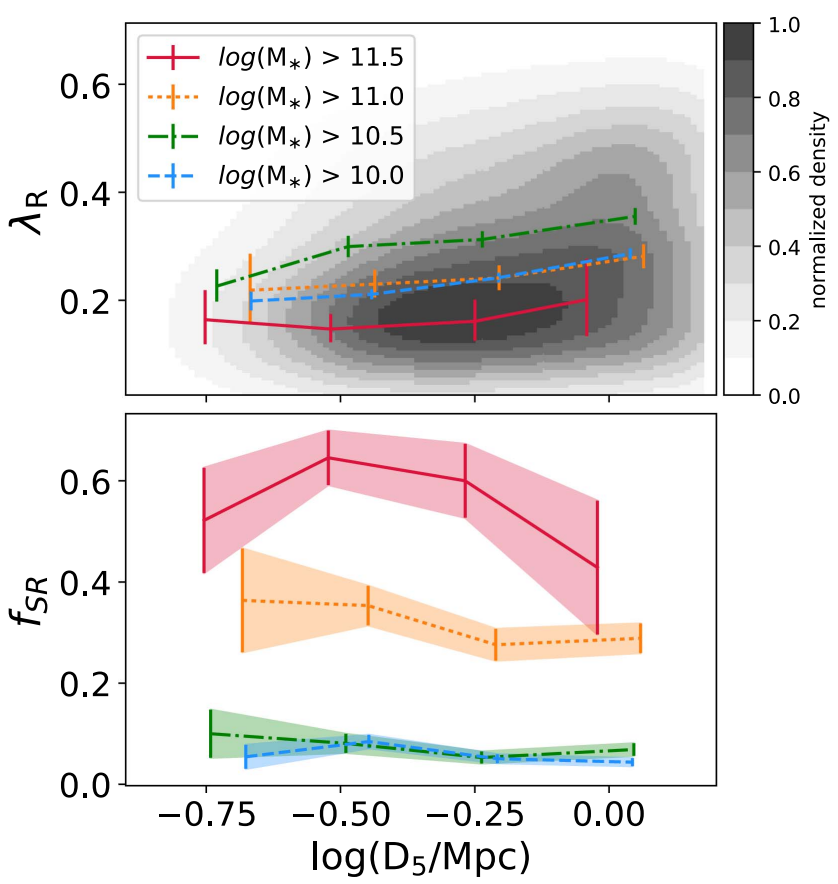

Figure 5. Dependence of $\lambda_{\mathrm{R}}$ and $f_{\mathrm{SR}}$ on the environmental density $D_{5}$. (a) Mean $\lambda_{R}$ of each mass bin with error bars indicating $95 \%$ confidence intervals of the mean from the bootstrap resampling on top of the $\lambda_{R}$ distribution (gray shade). (b) Slow rotator fraction in the same bin and the same color scheme as the top panel, with colored shading indicating the binomial error. Markers and error bars are at the mean $D_{5}$ in each bin.

agreement with the previous observational data of Brough et al. (2017) based on a cursory inspection of their Figure 11.

\subsection{The Varying Importance of Mergers}

In this section, we discuss the relative importance of mergers in each of the subgroups. In Figure 7, we show a 2D histogram of galaxies with a given number of merger events since $z=1$. Differences among merger counts of different subgroups are apparent. A large fraction of cluster centrals have undergone 2 to 5 major mergers (mass ratio $>1 / 4$ ) and 5 to 12 minor mergers (Figure $7(\mathrm{a})$ ). On the other hand, more than half $(62.7 \% ; 2075$ out of 3307$)$ of the cluster satellites have had no major mergers and at most one minor merger (Figure 7(b)). The majority $(86.3 \%$; 2855 out of 3307) of the cluster satellites fall within the four ( 2 by 2) bins with the lowest merger counts. Merger counts of the group centrals are between those of cluster centrals and cluster satellites (Figure 7(c)). In general, they undergo fewer mergers than cluster central ETGs, but undergo considerably more than cluster satellite ETGs. Field normal ETGs are concentrated within low merger count bins, but not as much as cluster satellite ETGs (Figure 7(d)). In summary, cluster centrals have the largest number of mergers among four subgroups, followed by group centrals, field ETGs, and then cluster satellite ETGs.

Given the dissimilar merger counts of the subgroups, we now revisit $\lambda_{\mathrm{R}}$ versus $\varepsilon$ in Figure 8. Group centrals predominantly occupy the low $\lambda_{\mathrm{R}}$ region with a considerable scatter in $\varepsilon$ (Figure 8(a)). The peak of cluster satellite distribution is in a low $\lambda_{\mathrm{R}}$ region, but there is a significant fraction stretching toward a higher $\lambda_{\mathrm{R}}$ and $\varepsilon$ region (Figure 8(b)). The difference in $f_{\mathrm{SR}}$ is significant at $58.1 \%$ (cluster centrals) versus $8.8 \%$ (cluster satellites). Finally, the group central ETGs show a larger scatter in the $\varepsilon$ direction and a lower mean $\lambda_{\mathrm{R}}$ compared to the field ETGs (Figures 8(c) and (d)).

Comparing Figures 7 and 8, a strong correlation between the number of mergers and the distribution in the $\lambda_{R}-\varepsilon$ plane is evident. First, the fact that most of the central galaxies have low spin and high $\varepsilon$ implies that mergers play an significant role in the kinematic evolution of cluster centrals after $z=1$ (e.g., Naab et al. 2014). By contrast, it is a rare occasion for cluster satellites to be disturbed by numerous mergers. Second, a 

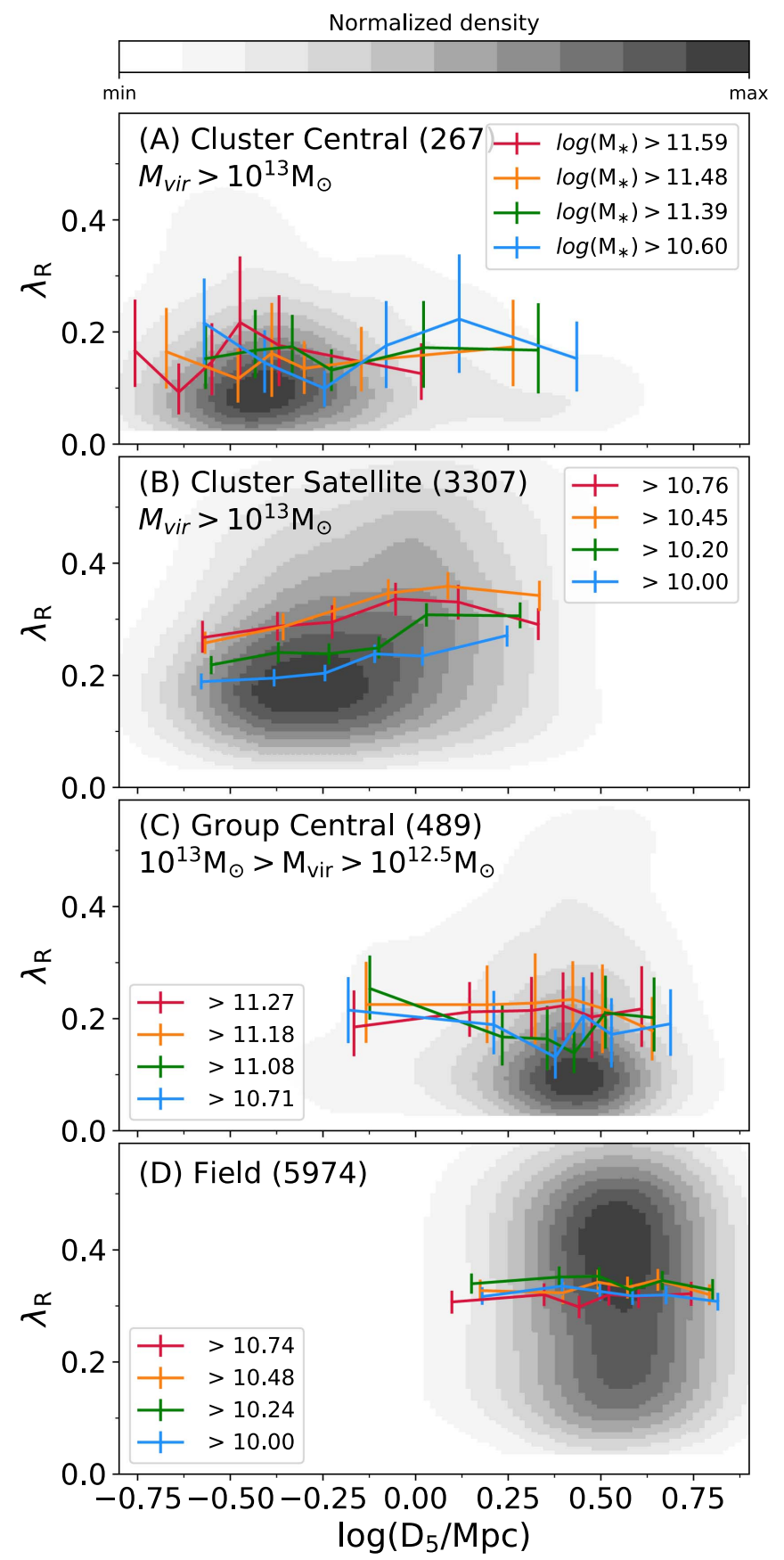

Figure 6. Similar to the top panel of Figure 5 but for each population of ETGs. In each panel, the samples are further divided into four subgroups of equal sample size. The background number density map is normalized within each panel, and error bars indicate $95 \%$ confidence intervals (CI) of mean from bootstrap resampling. No trends are found in panels (a), (c) and (d), whereas a mild trend is found in panel (b).

comparison between the $\lambda_{\mathrm{R}}$ distribution of cluster satellite and field ETGs reveals that the effect of a dense environment results in cluster satellite ETGs having a lower $\varepsilon$ with smaller scatter, as well as a lower mean $\lambda_{\mathrm{R}}$, which is in the opposite direction of the merger effects (Figures 8(b) and (d)).

\subsection{Mass Dependence by Subgroup}

In Figure 9, we revisit the galaxy spin divided in subgroups, focusing on the dependence on galaxy stellar mass and their host halo mass. This time, we combined cluster centrals and group centrals together because they share the same domain in the $\lambda_{\mathrm{R}}$ versus the stellar mass or the host halo mass plane.

We found that the mean $\lambda_{\mathrm{R}}$ trend of satellite ETGs is bent (see also Figure 4); less massive satellite ETGs rotate slower at the low-mass regime below $10^{11} M_{\odot}$, but more massive satellite ETGs rotate slower at the high-mass regime above $10^{11} M_{\odot}$ (Figure 9, middle panel). In the mass range of $10^{11} M_{\odot}<$ $M_{*}<3 \times 10^{11} M_{\odot}, \lambda_{\mathrm{R}}$ as well as $f_{\mathrm{SR}}$ of the satellite differ from that of the central galaxies, whereas they become similar above $3 \times 10^{11} M_{\odot}$. The strong mass dependence of satellite ETGs at the high-mass end can be explained by the very large cluster satellites that are, concurrently, central galaxies of groups, i.e., due to mergers.

Comparing satellite ETGs and field ETGs at the low-mass regime reveals some interesting results. The stellar mass distribution of field ETGs and satellite ETGs are similar, but the $\lambda_{\mathrm{R}}$ distributions are markedly different. The mean $\lambda_{\mathrm{R}}$ of field ETGs is nearly constant over the mass range, but the mean $\lambda_{\mathrm{R}}$ of the satellite ETGs clearly declines with decreasing stellar mass. The latter is directly the opposite of the negative mass dependence of spin of the most massive ETGs in groups, as previously seen from Figure 4 . Also note that $f_{\text {SR }}$ of field ETGs and cluster satellite ETGs are almost identical and constant below $M_{*} \simeq 10^{11} M_{\odot}$. At $\left\langle\lambda_{\mathrm{R}}\right\rangle \simeq 0.2$, cluster satellites at lower-mass end have a $f_{\mathrm{SR}}$ less than 0.1 , whereas cluster satellites at $M_{*} \approx 2 \times 10^{11}$ have $f_{\mathrm{SR}} \approx 0.25$. This implies that spin-down mechanism at the lower-mass end and higher-mass end are different.

Following the stellar mass dependence, galaxies embedded in sufficiently massive host halos $\left(M_{\mathrm{vir}}>10^{12.5} M_{\odot}\right)$ have comparable spins, regardless of whether they are satellites or centrals (Figure 9, right panels). For low-mass halos, the mean $\lambda_{\mathrm{R}}$ of satellites decreases with decreasing halo mass, whereas no clear trend is found in field ETGs. Although we have not included in this paper, we found that the halo mass distribution of field ETGs and cluster satellites is comparable at $z=1$, but the mass of satellite halos becomes smaller at $z=0$. This suggests that the galaxy spin down and the halo mass decrease occurred in conjunction.

We note that although 2 distinct mass dependencies are found in cluster satellites, $90 \%$ of the population fall in the lowmass regime below $M_{*}<10^{11}$. This implies that the environmental effects are influential for the spin evolution of the majority of satellite ETGs and that the merger-induced spin evolution, albeit stronger, is responsible for only small number of massive cluster satellite galaxies and central galaxies.

\subsection{Evolution of the Galaxy Spin}

Here, we move on to the time evolution of $\lambda_{\mathrm{R}}$. In Figure 10, we plot $\lambda_{\mathrm{R}}$ of sample galaxies and their main progenitors starting from $z=2$. We find that a significant fraction of cluster centrals at $z=2$ already rotate slowly (Figure 10(a)). By $z=1$, most of the cluster centrals rotate slowly at $\lambda_{\mathrm{R}}<0.2$, and the density peak at a low $\lambda_{\mathrm{R}}$ becomes more pronounced over time. Although the main progenitors of cluster satellite, group central, and field ETGs all initially rotate fast with density peaks at $\lambda_{\mathrm{R}} \approx 0.6$, their evolution tracks differ substantially.

Figure 10(b) illustrates a notable shift in the density peak of cluster satellite progenitors after around $z=0.4$. Eventually, the density peak moves toward $\lambda_{\mathrm{R}} \approx 0.2$ with a broad tail toward a higher $\lambda_{\mathrm{R}}$. The group central galaxies in Figure 10(c) 

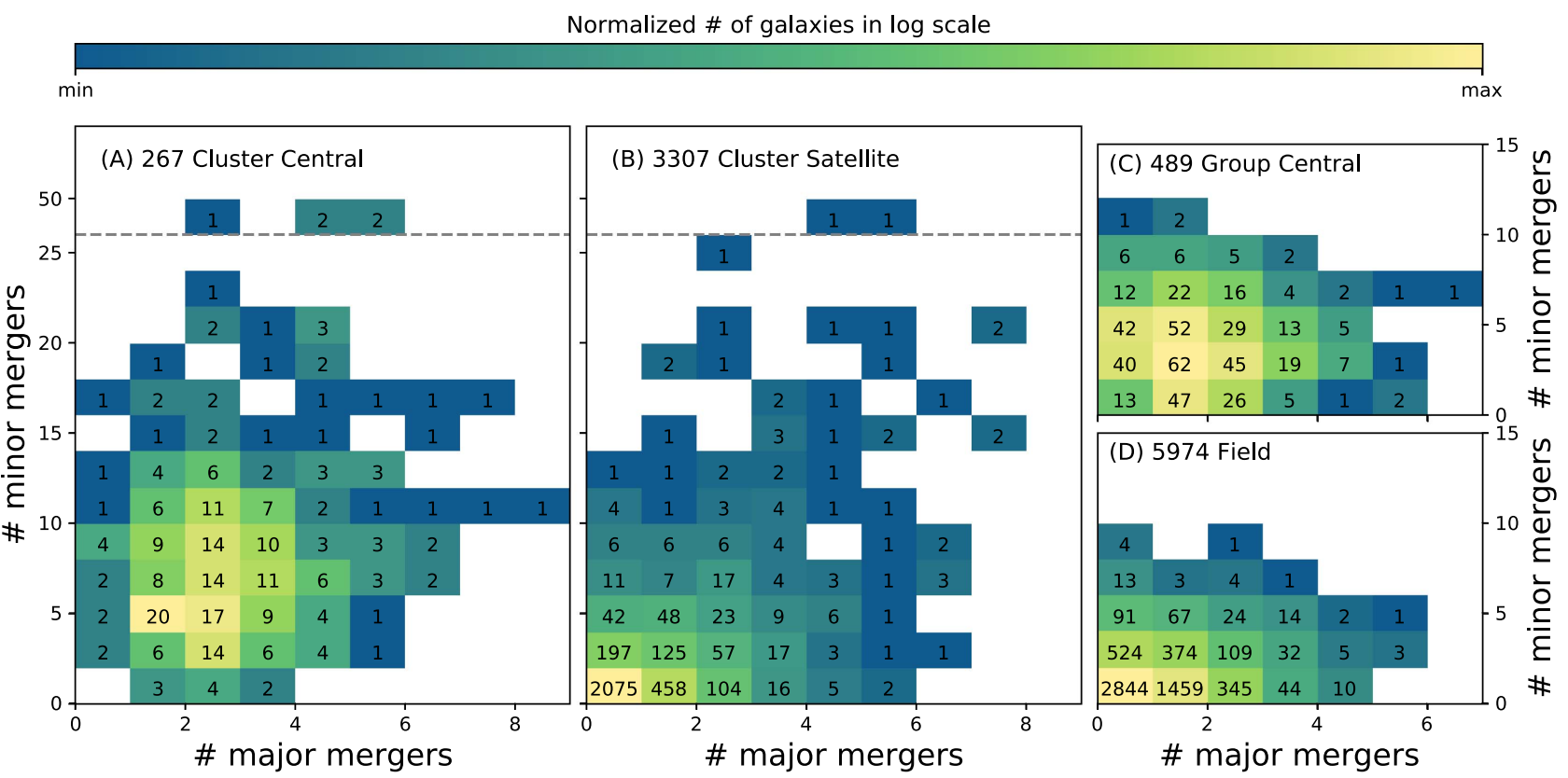

Figure 7. Numbers of major merging ( $x$-axis) and minor merging ( $y$-axis) galaxies since $z=1$, color-coded based on the relative number of galaxies in each subgroup. Group central ETGs (a) have the broadest peak at around 2-4 major mergers and 5-10 minor mergers. By contrast, a large fraction of cluster satellite ETGs (b) undergo no major or minor mergers. The peak at no merger is the narrowest among the four groups. Field central ETGs (c) form a peak at one major merger and two-four minor mergers. The peak is not at $(0,0)$ merger, but the overall merger count is slightly less than the group centrals. Field normal ETGs (d) have a peak at

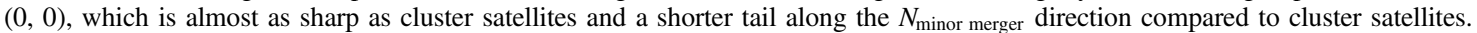

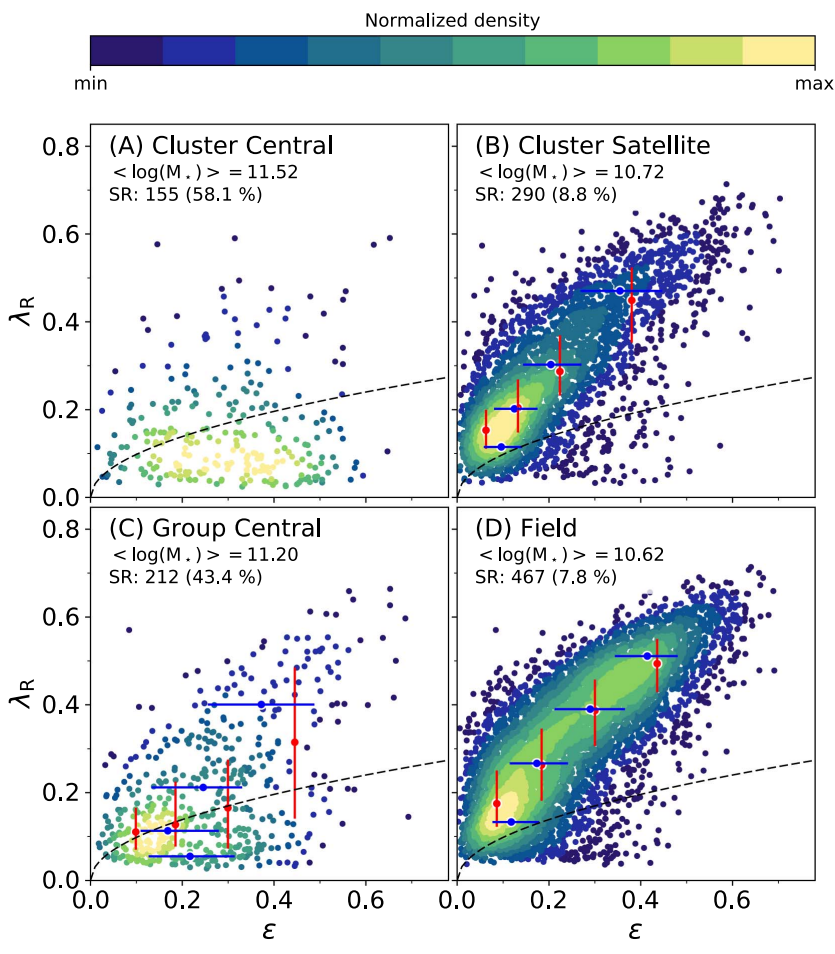

Figure 8. Spin parameter $\lambda_{\mathrm{R}}$ vs. ellipticity $\varepsilon$ as in Figure 3 for the cluster central (a), cluster satellite (b), group central (c), and field satellite (d) galaxies. Blue and red error bars represent $20 \%-80 \%$ range of $\varepsilon$ and $\lambda_{\mathrm{R}}$ independently binned along each axis.

demonstrate a noisy $\lambda_{\mathrm{R}}$ evolution track, with a peak at a very low value of $\lambda_{\mathrm{R}} \sim 0.1$ appearing since $z=0.5$. Without a merger or the effects from dense environments, field ETGs largely maintain their spin over time. Only a mild and gradual decline can been observed since $z=0.5$. Despite the fact that cluster satellites and field ETGs show comparable mean stellar masses and $\lambda_{\mathrm{R}}$ distributions at $z=2$, their $\lambda_{\mathrm{R}}$ evolution tracks are markedly dissimilar. Considering that cluster satellites undergo even fewer mergers than field ETGs, the larger spin down of cluster satellites represents the importance of their environment, not necessarily mergers.

Next, we present the relative importance of mergers in galaxy spin evolution. Specifically, we measured the absolute $\lambda_{R}$ change between adjacent snapshots and determined if there were merger events taking place simultaneously. If a galaxy was affected by one or more mergers continuously since $z=2.0$, we determined the merger contribution to the spin change to be $100 \%$. However, this does not necessarily imply that the physical origin of the $\lambda_{R}$ change is entirely the result of mergers because other concurrent mechanisms could be operating. Hence the results refer only to the upper limit of the merger impact. Even as an upper limit, the majority of the satellite galaxies are only marginally influenced by merger events, mainly due to the lack of galaxy mergers (Figure 11). In contrast, as discussed in the previous section, mergers appear to be largely responsible for the spin change in the cluster central and group central ETGs.

\subsection{Non-merger Tidal Interactions}

The gravitational potential of cluster/group environments leads to low probabilities of galaxy mergers, but a higher likelihood of high-speed encounters. Hydrodynamical processes, such as ram pressure stripping, or strangulation, are also important aspects of the environmental effects in clusters; however, they do not seem to directly perturb the stellar structure (e.g., Smith et al. 2012) and can only passively influence the galaxy properties by removing cold gas and shutting down new star formation. Although forming new stars from late accretion is a possible way to spin-up ETGs (Lagos et al. 2017; Penoyre et al. 2017), star formation is insignificant 

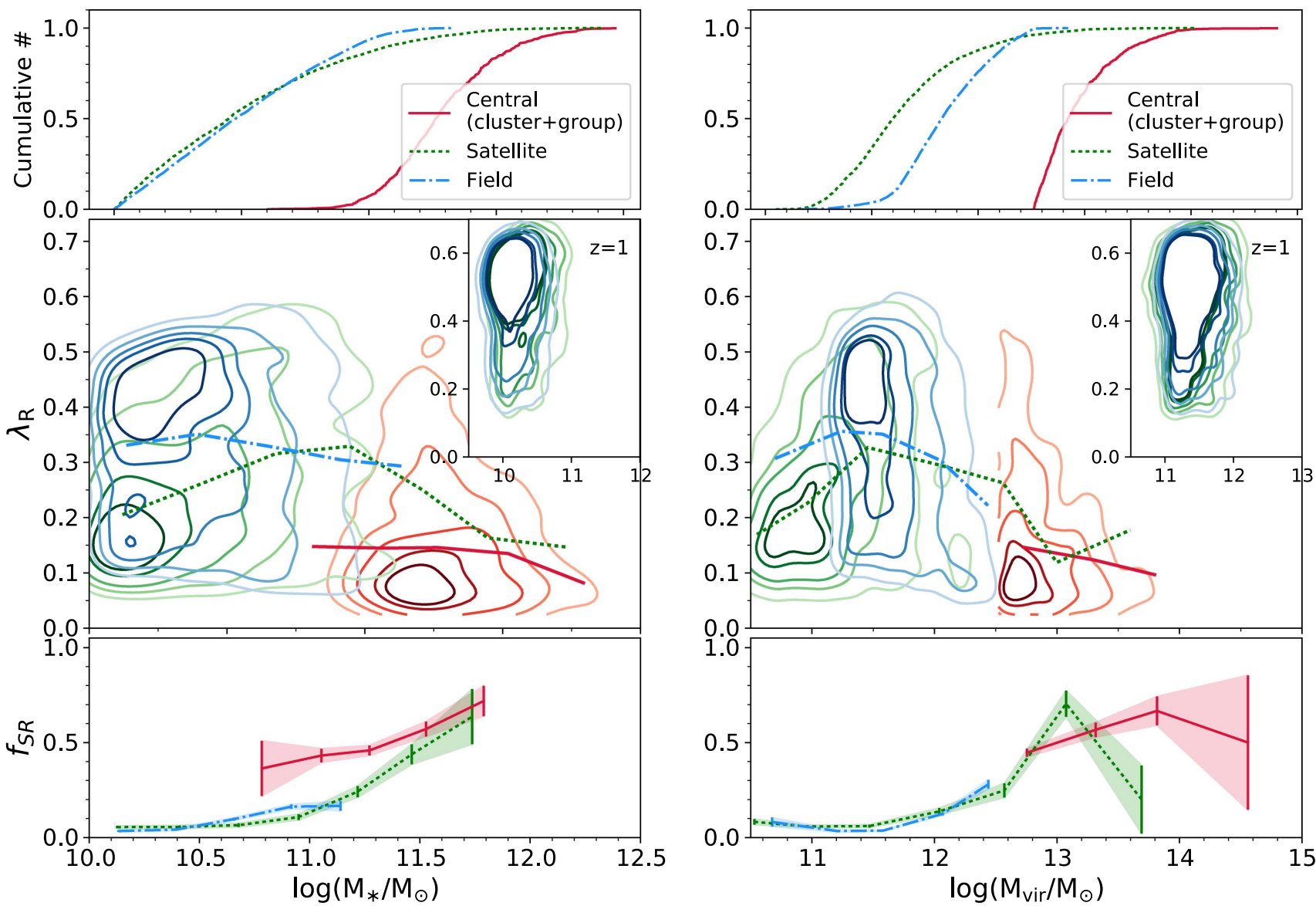

Figure 9. Top: cumulative mass function of galaxies normalized for each subgroup. Middle: $\lambda_{\mathrm{R}}$ dependency on stellar mass (left) and host halo mass (right), for each subgroup. (Bottom) Slow rotator fraction $f_{\mathrm{SR}}$ plotted as a function of mass for each subgroup. The mean galaxy spin $\lambda_{\mathrm{R}}$ of the central, satellite, and field ETGs is indicated by the solid red line, dotted green line, and dotted-dashed blue line, respectively. Each contour line encompasses 10\%, 20\%, 40\%, 60\%, and 80\% of each sample, respectively. Note that cluster central and group central galaxies are combined in this plot for the purposes of clarity. Each inset panel shows the distributions of the progenitors of field and satellite galaxies at $z=1$ in the same color scheme, contour levels, and axes as the middle panel. Because two subgroups are split by host halo masses, and their stellar masses roughly correlate with their host halo masses, it can be assumed that two-thirds of the central galaxies at the lower-mass end are group central ETGs.

in both the cluster central and field ETG samples, and thus we focused on tidal effects in our analysis.

To quantify the cumulative tidal effects on each satellite galaxy through non-merger encounters, we used the perturbation index (PI) inspired by Byrd \& Valtonen (1990), which is defined as:

$$
\mathrm{PI}=\log \left[\int_{t_{0}}^{t} \sum_{i=0}^{n}\left(\frac{M_{p, i}}{M_{\mathrm{gal}}}\right) \times\left(\frac{R_{\mathrm{gal}}}{d_{p, i}}\right)^{3} d t / \mathrm{Gyr}\right],
$$

where $M_{\text {gal }}$ and $R_{\text {gal }}$ are the mass and size of the galaxy in question, $M_{p, i}$ and $d_{p, i}$ are the mass and distance to the $i$ th perturber, respectively, and the time integration runs between two epochs in Gyr unit.

Any galaxy exceeding $5 \times 10^{8} M_{\odot}$ within $2 h^{-1} \mathrm{Mpc}$ from the galaxy in question is incorporated into the summation. Thanks to the power of -3 dependence on the distance, the PI quickly converges at a scale of few hundreds of kpc.

The PI is primarily designed to measure the tidal effect of the global potential field, but is also sensitive to galaxy harassment and galaxy mergers (one major merger corresponds to $\mathrm{PI} \approx \log 1=0$ ). Thus, in this and the following sections, we focus our discussion on galaxies that have undergone no major or minor mergers since $z=1$. This strict criterion leaves $47 \%$ (1520) of cluster satellites and 31\% (1767) of field ETGs. We checked that including galaxies with up to 3 minor mergers (leaving 69\% and 56\% of each group, respectively) in the analysis did not affect the main results.

In Figure 12, we compared the amount of the spin change against the PI of cluster satellite and field ETGs without mergers since $z=1$. The cluster satellites form a peak at $\mathrm{PI} \simeq-1$ and $\Delta \lambda_{\mathrm{R}} \simeq-0.25$ in a triangular-shaped envelop (Figure 12(a)). Although the scatter in $\Delta \lambda_{R}$ intensifies with an increasing PI, galaxies with a larger PI show a larger drop in $\lambda_{\mathrm{R}}$ since $z=1$. It is also interesting to see that most galaxies with a low PI $(\sim-3)$ are clustered around a small $\Delta \lambda_{\mathrm{R}}(\sim-0.1)$, meaning that it is difficult for a galaxy to lose its spin significantly without being affected by either mergers or environmental tidal effects. By contrast, field ETGs are only minimally perturbed (PI $\lesssim-2$; Figure $12(\mathrm{~b})$ ). This indicates that the distributions of cluster satellite and field ETGs are almost mutually exclusive (Figure 12(c)). Given that the mean stellar mass, mean $\lambda_{R}$, and mean specific star formation rate (sSFR) of satellite galaxies and field ETGs are comparable at $z \simeq 1$ (Figure 13), the difference in their evolution suggests that environmental differences play a major role in determining the galaxy spin in the absence of mergers. 


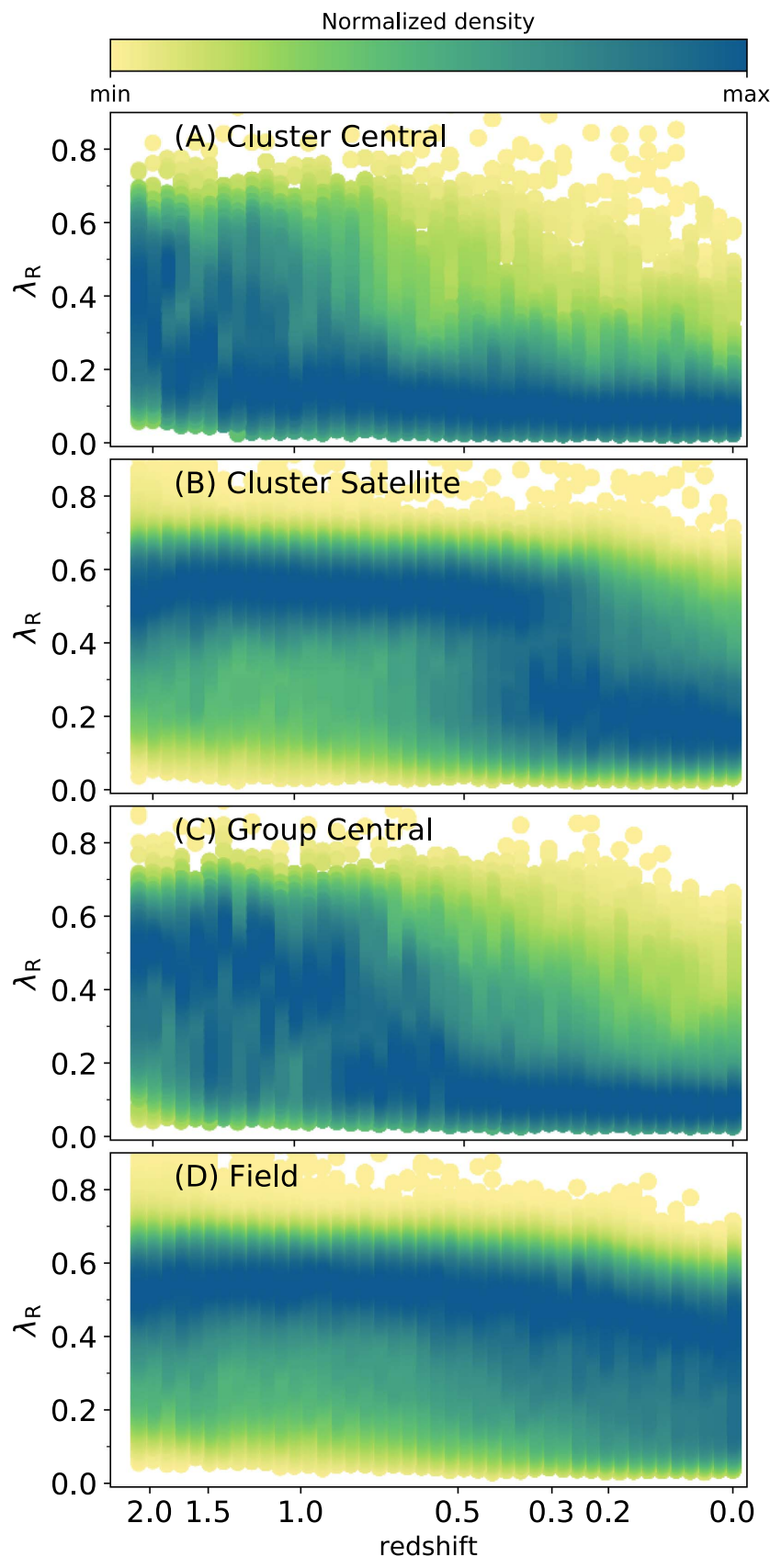

Figure 10. Evolution of $\lambda_{\mathrm{R}}$ in subsamples of different environments since $z=2$. We followed the main progenitor of each galaxy. The $x$-axis, representing the redshift, is linear in physical time.

\subsection{A Scenario of Satellite ETG Evolution}

A more thorough picture of the spin evolutions of cluster satellite ETGs in comparison with field counterparts is presented in Figure 13. Again, we only consider galaxies with no merger events since $z=1$ for a more robust comparison. We plot the evolution of the mean PI of cluster satellites and field ETGs in orange and blue, respectively, and 25\%-75\% ranges through shadings. Albeit it is the large scatter, the typical PIs of cluster satellites and field ETGs are comparable in the early epoch $(z \sim 2)$, but they begin to deviate at $z \simeq 1$, as only cluster satellites are continually perturbed by interactions with nearby galaxies (Figure 13(a)). In other words, the environmental difference of the two populations becomes noticeable at $z \simeq 1$.

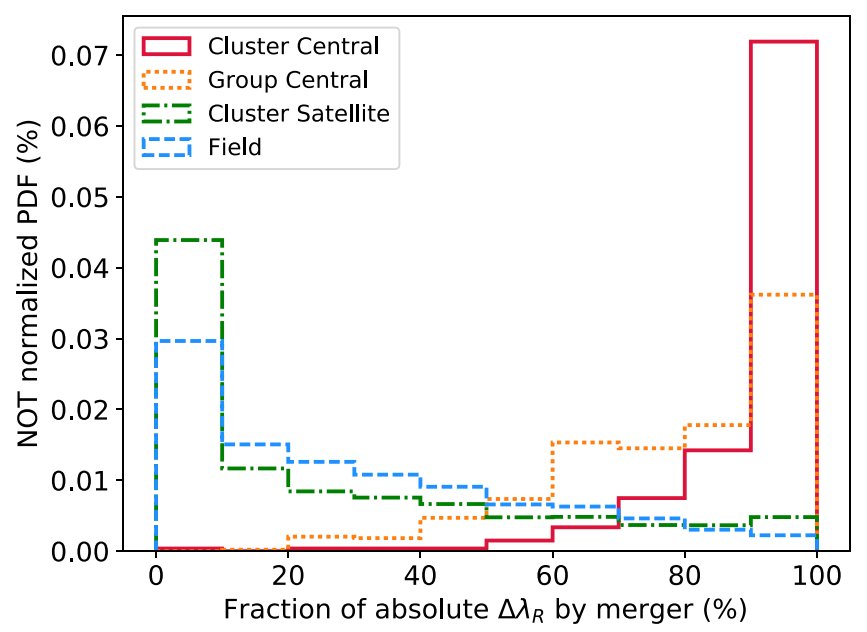

Figure 11. Histogram of the fraction of absolute $\lambda_{\mathrm{R}}$ change between snapshots occurred during merger events since $z=2$. Note that a galaxy with a $100 \%$ fraction means that it is continuously affected by one or more mergers since $z=2$. We show the absolute value to highlight the ability of mergers to make changes in the galaxy spin in any direction.

In Figure 13(b), we plot the fractional mass change of galaxies compared to the mass at $z=0.5$, after which the environmental effects accelerate the deviation in the properties of the cluster satellites and the field ETGs. The mean stellar masses of the two populations at $z=2$ are $5.7 \times 10^{9} M_{\odot}$ and $7.4 \times 10^{9} M_{\odot}$, respectively. Contrary to the persistent stellar mass growth of field ETGs until $z=0$, the stellar mass of cluster satellites grows more slowly and eventually decreases after $z=0.2$. The trends in SSFR (Figure 13(c), measured over the previous $0.1 \mathrm{Gyr}$ ) and $\lambda_{\mathrm{R}}$ (Figure 13(d)) are similar in that cluster satellite ETGs initially have comparable values to the field ETGs, but ultimately have lower values than field ETGs at $z=0$, which is probably due to the ram pressure stripping of cold gas in the former case.

To highlight the differential evolution of cluster satellite and field ETGs, we plot the fractional residual of each quantity $(q)$ in Figure 13(e), which is defined as $f_{q} \equiv\left\langle q_{\mathrm{GS}}\right\rangle /\left\langle q_{\mathrm{FN}}\right\rangle-1$, where GS and FN represent cluster satellites and field ETGs, respectively. Note that we plot the residual fraction of the absolute stellar mass $\left(=\left\langle M_{*, \mathrm{GS}}\right\rangle /\left\langle M_{*, \mathrm{FN}}\right\rangle-1\right)$, instead of the fractional change in the stellar mass, as in Figure 13(b), for a valid comparison. The initial properties of cluster satellite and field ETGs are comparable, except for the $25 \%$ difference in the mean stellar mass. Yet different properties seem to evolve at different rates. The PI of cluster satellites begin to rise significantly at $z \simeq 1$, accompanied by the slow increase in stellar mass, followed by a reduction in $\lambda_{\mathrm{R}}$ after $z \simeq 0.5$.

It is also worth noting that the decline in the sSFR lags behind the rise or fall in the rest of the properties. This suggests that tidal perturbations initiate the spin down of satellite ETGs, whereas star formation quenching due to environmental effects may help to maintain the slow rotation at the later stages.

\section{Discussion and Conclusion}

We analyzed ETGs more massive than $10^{10} M_{\odot}$ from the $\left(100 h^{-1} \mathrm{Mpc}\right)^{3}$ volume of the Horizon-AGN simulation. The simulated ETGs reasonably reproduced the loci of $\lambda_{R}$ versus the $\varepsilon$ distribution from IFU observations (Emsellem et al. 2007; Cappellari et al. 2011). We found an increase in $f_{\mathrm{SR}}$ along the 

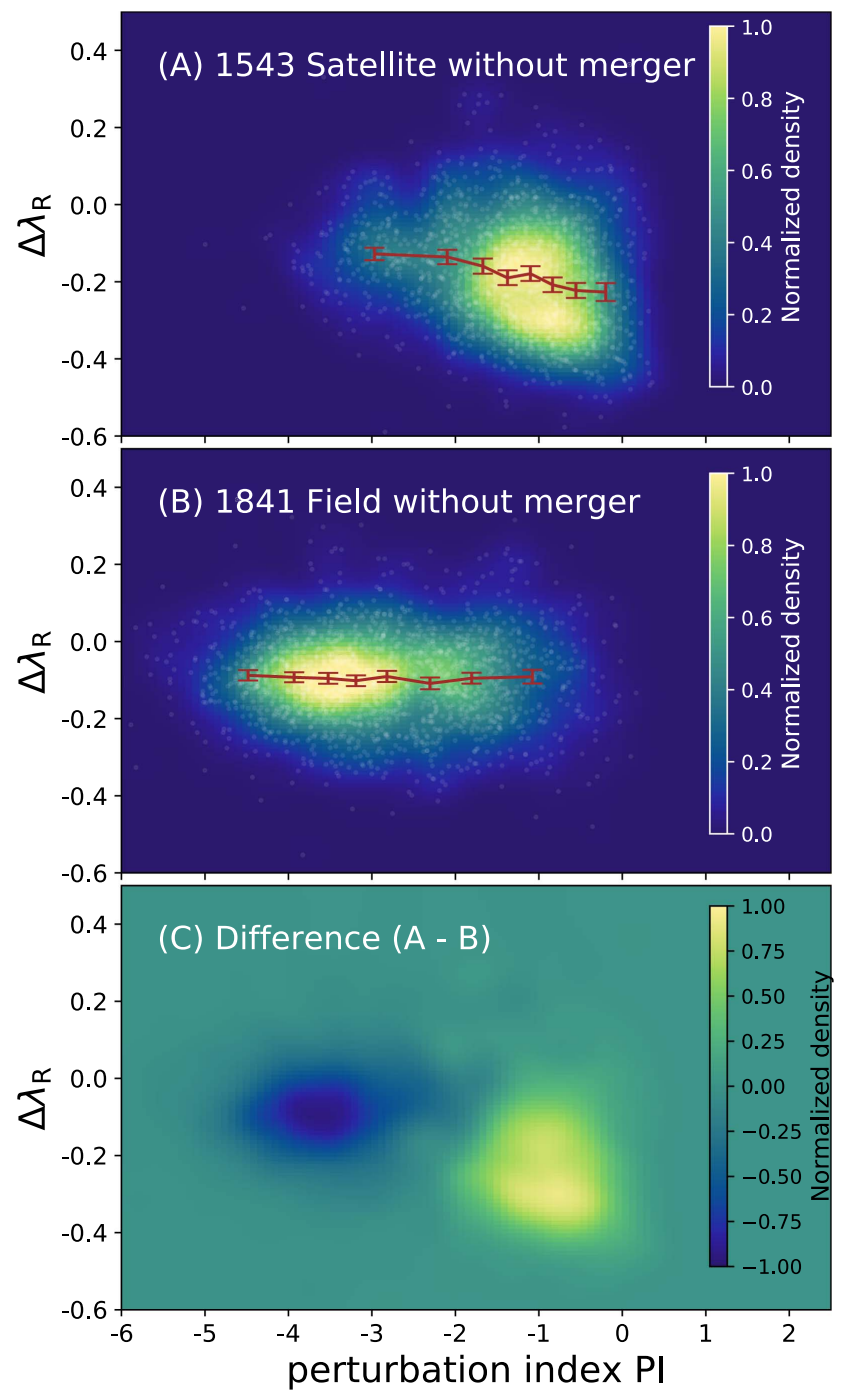

Figure 12. Change in $\lambda_{\mathrm{R}}$ from $z=1$ to $z=0$ of satellite galaxies (a) and field ETGs (b), and the difference between the two populations (c). Only galaxies without mergers are considered in this plot. The color scale is identical across all three panels, representing normalized height according to the peak in panel (a). A considerable fraction of field ETGs have undergone negligible tidal interactions, whereas a large fraction of satellites are severely affected by tidal interactions and lose their spin by more than $\Delta \lambda_{R} \simeq-0.2$. Red error bars indicate $95 \%$ confidence intervals of the mean from bootstrap resampling.

increasing stellar mass within a mass range of above $\sim 5 \times 10^{10} M_{\odot}$, which is in agreement with recent observations by D'Eugenio et al. (2013), Houghton et al. (2012), Fogarty et al. (2014), Cappellari (2016), Veale et al. (2017), Brough et al. (2017), and Greene et al. (2018). While the marginal correlation between the mean $\lambda_{\mathrm{R}}$ and the environmental density of the total ETG sample is consistent with the observations, varying dependencies of the ETG spin on the stellar mass and environments were found when ETGs are divided into subgroups.

Having evolved through numerous mergers, more massive (both group and field) central ETGs rotate more slowly, regardless of their environment. This clear mass dependence of the spin of central ETGs is consistent with the aforementioned IFU observations and can be explained by the merger-driven formation scenario (Naab et al. 2014; Lagos et al. 2017; Penoyre et al. 2017). Field normals, with fewer mergers than central ETGs, more or less have maintained their $\lambda_{R}$ throughout
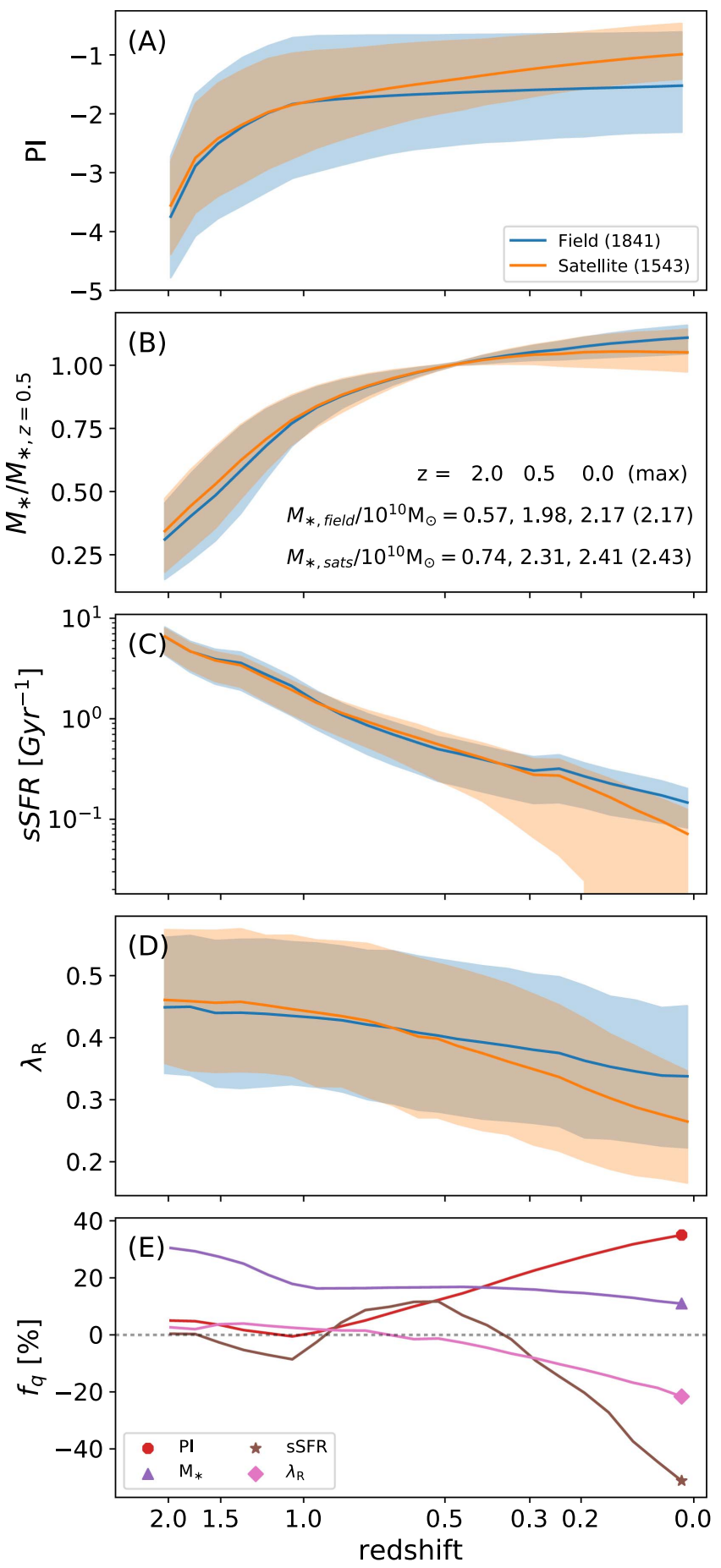

Figure 13. Evolutionary trajectories of the mean properties of 1543 cluster satellites and 1841 field counterparts without mergers since $z=1$. (a) Perturbation index. (b) Fractional change in the stellar mass with respect to the stellar mass at $z=0.5$. (c) Specific star formation rate. (d) Galaxy spin. (e) Fractional residuals of cluster satellite properties with respect to those of field ETGs. Shaded regions indicate the upper and lower quartiles (25\%-75\%) of each distribution, and solid lines represent the mean values. The $x$-axis, representing the redshift, is linear in physical time. Note that the sudden drop of the lower envelop occurs when the bottom $25 \%$ of the satellites have an undetectable star formation (no new stellar particles). In panel (e), unlike in panel (b), we plot the fraction of the difference in the stellar mass $\left(\left[M_{*, \mathrm{GS}}-M_{*, \mathrm{FN}}\right] / M_{*, \mathrm{FN}}\right)$.

the cosmic evolution, showing no dependence on stellar mass or environments. Interestingly, satellite ETGs in massive halos rotate much slower than field ETGs at similar stellar masses, 
even though satellites undergoes fewer mergers than their field counterparts.

We demonstrated the importance of environmental tidal effects by comparing satellite ETGs and field ETGs whose progenitors shared similar properties until $z \simeq 1$. The satellite ETGs that have suffered from a higher degree of tidal perturbation since $z \simeq 1$ showed a larger drop in their spin. By contrast, field ETGs accumulated only negligible amount of tidal effects and showed correspondingly small $\Delta \lambda_{\mathrm{R}}$, unless they have undergone mergers. A clear separation is made between satellite ETGs and field ETGs without mergers on the PI (Equation (3)) versus the $\Delta \lambda_{\mathrm{R}}$ plane, which is caused by the differential environments after $z \simeq 1$.

Consequently, the $\lambda_{\mathrm{R}} \mathrm{s}$ of the satellite ETGs show distinct features because their spin evolution, with the absence of mergers, is mainly determined by the cumulative effect of tidal perturbation, as quantified by PI. First, the mass dependence of $\lambda_{\mathrm{R}}$ of the satellite ETGs is not monotonic. The mean $\lambda_{\mathrm{R}}$ of the satellite ETGs below $M_{*}<10^{11} M_{\odot}$ has a positive mass dependence, which is the opposite to the negative dependence of central ETGs in dense environments. This outcome is likely because, among satellites, smaller ETGs are more susceptible to their environments and thus lose spin more easily. Second, a clear environmental dependence of the mean $\lambda_{\mathrm{R}}$ is visible: cluster satellites in dense regions have lower mean $\lambda_{R} \mathrm{~s}$ than those in a less dense region.

Our selection criterion of ETGs involves possible contamination from LTGs (Section 2.4). Even so, we argue that a precise distinction between relatively slow-rotating LTGs and relatively fast-rotating ETGs is unimportant in our analysis, considering the fact that galaxy properties are continuous functions of galaxy spin (Cappellari 2016). Nonetheless, we confirmed that adopting an alternative criterion (for example, that of Schawinski et al. 2014) did not change our main conclusions.

Galaxy mergers are indeed prevalent at high redshifts $(z \gtrsim 2)$, but at lower redshifts, they are the dominant driver of the spin down only for central ETGs in dense regions, which represent less than $10 \%$ of the whole ETGs population. By contrast, environmental perturbation drives the slower rotation of cluster satellites compared to their field counterparts. The details of our model prediction is, at the moment, more than challenging to test through current observational techniques. Nonetheless, we expect that future observations using the Multi Unit Spectroscopic Explorer (MUSE; Bacon et al. 2010) or Hector (Bryant et al. 2016) may be able to test our scenario by constructing a more extensive catalog over a wider stellar mass range.

S.K.Y., acting as the corresponding author, acknowledges support from the Korean National Research Foundation (NRF-2017R1A2A1A05001116). This study was performed under the umbrella of the joint collaboration between the Yonsei University Observatory and the Korea Astronomy and Space Science Institute. The analysis were carried on the Horizon Cluster hosted by Institut dAstrophysique de Paris. We thank $\mathrm{S}$. Rouberol for running it smoothly for us. This research is part of Spin(e) (ANR-13-BS05-0005, http://cosmicorigin.org).

\section{ORCID iDs}

Hoseung Choi (iD https://orcid.org/0000-0001-7229-0033 Sukyoung K. Yi (ib https://orcid.org/0000-0002-4556-2619 Taysun Kimm (iD https://orcid.org/0000-0002-3950-3997

\section{References}

Abadi, M. G., Navarro, J. F., Steinmetz, M., \& Eke, V. R. 2003, ApJ, 597, 21 Aubert, D., Pichon, C., \& Colombi, S. 2004, MNRAS, 352, 376

Bacon, R., Accardo, M., Adjali, L., et al. 2010, Proc. SPIE, 7735, 773508 Barnes, J. E. 1988, ApJ, 331, 699

Bois, M., Emsellem, E., Bournaud, F., et al. 2011, MNRAS, 416, 1654 Bondi, H., \& Hoyle, F. 1944, MNRAS, 104, 273

Boselli, A., Boissier, S., Cortese, L., \& Gavazzi, G. 2009, AN, 330, 904

Boselli, A., Voyer, E., Boissier, S., et al. 2014, A\&A, 570, A69

Bottrell, C., Torrey, P., Simard, L., \& Ellison, S. L. 2017, MNRAS, 467, 2879

Brough, S., van de Sande, J., Owers, M. S., et al. 2017, ApJ, 844, 59

Bruzual, G., \& Charlot, S. 2003, MNRAS, 344, 1000

Bryant, J. J., Bland-Hawthorn, J., Lawrence, J., et al. 2016, Proc. SPIE, 9908 , $99081 \mathrm{~F}$

Byrd, G., \& Valtonen, M. 1990, ApJ, 350, 89

Cappellari, M. 2016, ARA\&A, 54, 597

Cappellari, M., \& Copin, Y. 2003, MNRAS, 342, 345

Cappellari, M., Emsellem, E., Krajnović, D., et al. 2011, MNRAS, 413, 813

Cappellari, M. M. 2002, MNRAS, 333, 400

Choi, H., \& Yi, S. 2017, ApJ, 837, 1

Dekel, A., Sari, R., \& Ceverino, D. 2009, ApJ, 703, 785

D’Eugenio, F., Houghton, R. C. W., Davies, R. L., \& Dalla Bontà, E. 2013, MNRAS, 429, 1258

Di Matteo, P., Combes, F., Melchior, A.-L., \& Semelin, B. 2007, A\&A, 468, 61 Dressler, A. 1980, ApJ, 236, 351

Dubois, Y., Devriendt, J., Slyz, A., \& Teyssier, R. 2012, MNRAS, 420, 2662

Dubois, Y., Pichon, C., Welker, C., et al. 2014, MNRAS, 444, 1453

Dubois, Y., \& Teyssier, R. 2008, A\&A, 477, 79

Emsellem, E., Cappellari, M., Krajnović, D., et al. 2007, MNRAS, 379, 401 Emsellem, E., Cappellari, M., Krajnović, D., et al. 2011, MNRAS, 414, 888 Emsellem, E., Monnet, G., Bacon, R., \& Nieto, J.-L. 1994, A\&A, 285, 739 Fogarty, L. M. R., Scott, N., Owers, M. S., et al. 2014, MNRAS, 443, 485

Gabor, J. M., Davé, R., Finlator, K., \& Oppenheimer, B. D. 2010, MNRAS, 407,749

Greene, J. E., Leauthaud, A., Emsellem, E., et al. 2017, ApJL, 851, L33

Greene, J. E., Leauthaud, A., Emsellem, E., et al. 2018, ApJ, 852, 36

Guérou, A., Emsellem, E., Mcdermid, R. M., et al. 2015, ApJ, 804, 70

Haardt, F., \& Madau, P. 1996, ApJ, 461, 20

Houghton, R. C., Davies, R. L., Dalla Bontà, E., \& Masters, R. 2012, MNRAS, 423, 256

Hoyle, F., \& Lyttleton, R. A. 1939, MPCPS, 35, 592

Janz, J., Penny, S. J., Graham, A. W., Forbes, D. A., \& Davies, R. L. 2017, MNRAS, 468, 2850

Jimmy, Tran, K.-V., Brough, S., et al. 2013, ApJ, 778, 171

Kaviraj, S., Laigle, C., Kimm, T., et al. 2017, MNRAS, 467, 4739

Kennicutt, R. C., Jr. 1998, ApJ, 498, 541

Khim, H.-g., Park, J., Seo, S.-W., et al. 2015, ApJS, 220, 3

Khochfar, S., Emsellem, E., Serra, P., et al. 2011, MNRAS, 417, 845

Komatsu, E., Smith, K. M., Dunkley, J., et al. 2011, ApJS, 192, 18

Lagos, C. d. P., Stevens, A. R. H., Bower, R. G., et al. 2018, MNRAS, 473, 4956

Lagos, C. d. P., Theuns, T., Stevens, A. R., et al. 2017, MNRAS, 464, 3850

Naab, T., Oser, L., Emsellem, E., et al. 2014, MNRAS, 444, 3357

Oliva-Altamirano, P., Brough, S., Tran, K.-v., et al. 2017, AJ, 153, 89

Penoyre, Z., Moster, B. P., Sijacki, D., \& Genel, S. 2017, MNRAS, 468, 3883

Rasera, Y., \& Teyssier, R. 2006, A\&A, 445, 1

Sales, L. V., Navarro, J. F., Theuns, T., et al. 2012, MNRAS, 423, 1544

Salpeter, E. E. 1955, ApJ, 121, 161

Scannapieco, C., White, S. D. M., Springel, V., \& Tissera, P. B. 2009, MNRAS, 396, 696

Schawinski, K., Urry, C. M., Simmons, B. D., et al. 2014, MNRAS, 440, 889 Scott, N., Davies, R. L., Houghton, R. C. W., et al. 2014, MNRAS, 441, 274 Skibba, R. A., Bamford, S. P., Nichol, R. C., et al. 2009, MNRAS, 399, 966 Smith, R., Fellhauer, M., \& Assmann, P. 2012, MNRAS, 420, 1990

Sutherland, R. S., \& Dopita, M. A. 1993, ApJS, 88, 253

Teyssier, R. 2002, A\&A, 385, 337

Toloba, E., Guhathakurta, P., Boselli, A., et al. 2015, ApJ, 799, 172

Toomre, A., \& Toomre, J. 1972, ApJ, 178, 623

Tweed, D., Devriendt, J., Blaizot, J., Colombi, S., \& Slyz, A. 2009, A\&A, 506, 647

van de Sande, J., Bland-Hawthorn, J., Brough, S., et al. 2017a, MNRAS, 472,1272

van de Sande, J., Bland-Hawthorn, J., Fogarty, L. M. R., et al. 2017b, ApJ, 835,104

Veale, M., Ma, C.-P., Greene, J. E., et al. 2017, MNRAS, 471, 1428

Vollmer, B. 2009, A\&A, 502, 427 